\title{
Fertility preservation in childhood and adolescent female tumor survivors.
}

\author{
Francesca FILIPPI, M.D. ${ }^{1}$; Cristina MEAZZA, M.D. ${ }^{2}$; Edgardo SOMIGLIANA, M.D. Ph.D. ${ }^{1,3}$ *, \\ Marta PODDA, M.D. ${ }^{2}$; Chiara DALLAGIOVANNA, M.D. ${ }^{1,3}$; Maura MASSIMINO, M.D. ${ }^{2}$, \\ Francesco RASPAGLIESI, M.D. ${ }^{4}$; Monica TERENZIANI, M.D. ${ }^{2}$
}

${ }^{1}$ Fondazione IRCCS Ca' Granda Ospedale Maggiore Policlinico, Milan, Italy

${ }^{2}$ Pediatric Oncology Unit, Fondazione IRCCS Istituto Nazionale Tumori, Milan, Italy

${ }^{3}$ Dept of Clinical Sciences and Community Health, Università degli Studi di Milano, Milan, Italy

${ }^{4}$ Gynecological Oncology Unit, Fondazione IRCCS Istituto Nazionale Tumori, Milan, Italy

* To whom correspondence should be addressed

Edgardo SOMIGLIANA

Infertility Unit, Fondazione IRCCS Ca' Granda Ospedale Maggiore Policlinico

Via M. Fanti, 6 - 20122 - Milan - Italy

Phone: +39-02-55034304 Fax: +39-02-55036581

Email: dadosomigliana@yahoo.it

Authors financial disclosure: None

Financial support: None

Conflict of interests: Dr. Somigliana reports grants from Ferring, grants and personal fees from Merck-Serono, grants and personal fees from Theramex, outside the submitted work. All the other authors do not have any Conflict of interest to declare. 


\section{Capsule}

Ovarian reserve impairment is common in childhood and adolescence female tumor survivors. Oocytes cryopreservation may deserve consideration in this setting. 


\section{AbSTRaCT}

Objectives: To assess the proportion of childhood and adolescent female tumor survivors who could benefit from oocytes cryopreservation.

Design: Case series of childhood and adolescence female tumor survivors referred for fertility counseling.

Setting: A referral Cancer Center and an infertility Unit of an academic hospital.

Patients: Young childhood and adolescent female tumor survivors who received gonadotoxic treatments.

Interventions: Women were prescribed tests of ovarian reserve and a personalized counseling was given. Oocytes cryopreservation was considered in subjects aged $\geq 18$ years who were diagnosed with diminished ovarian reserve (DOR) $(\mathrm{AMH}<2 \mathrm{ng} / \mathrm{ml}$ or total $\mathrm{AFC} \leq 10$.

Main Outcome Measure: Rate of women with DOR who stored their oocytes.

Results: Ninety out of 126 evaluated women completed the assessments. We documented preserved ovarian reserve, DOR and premature ovarian insufficiency in 36 (40\%), 35 (39\%) and 19 (21\%) cases, respectively. Overall, 13 subjects with DOR were eligible for oocytes cryostorage, of whom nine $(69 \%)$ underwent the procedure. Considering the whole cohort of evaluated young women $(n=90)$, the rate of those who performed egg freezing was $10 \%$. Finally, nine women started looking for pregnancy after the counseling (six with DOR) and seven of them became pregnant. When data were analyzed separately according to most gonadotoxic treatments, considerable differences emerged but evidence did not support the idea that counseling should be restricted to particular subgroups of women.

Conclusions: Ovarian reserve impairment is common in childhood and adolescence female tumor survivors. Post-cancer oocytes cryopreservation may be part of the armamentarium of fertility preservation options. 
KEY WORDS: childhood tumor survivor; fertility preservation; oocytes cryopreservation; ovarian reserve 


\section{Introduction}

Oncofertility care is not limited to fertility preservation discussion and treatments at the time of cancer diagnosis. Patient care should include management of hormonal and sexual dysfunction, contraception and fertility counseling, both during and after cancer treatment (1-5).

Childhood and adolescent female survivors represent a peculiar population, for whom gynecological assessment and fertility counseling after the end of cancer treatment may be particularly important $(4,6-8)$. Of further relevance here is that fertility preservation issues are generally discussed more in depth with parents at the time of cancer diagnosis rather than with the patients themselves, in particular for the youngest children. Even if it is recommended to involve children in fertility discussion since the age of seven years, information in young patients is tailored to the patient age and it is inevitably incomplete and simplistic $(2,9)$. Many survivors have anchored and biased beliefs about their fertility status or report frustration due to the lack of reproductive continuity of care $(2,10)$. Importantly, in most cases no fertility preservation measures could be adopted at the time of cancer diagnosis because of unsuitable clinical conditions or because parents declined. Post-cancer oocytes cryopreservation is an emerging option for these patients but evidence is extremely scanty $(11,12)$.

In general, survivors of children and adolescent cancers represent a neglected group with critical but unmet needs (8). For this reason, we implemented a systematic referral of all childhood and adolescent tumor survivors to the oncofertility service with the intent to provide individual fertility assessment and pro-fertility counseling. The present study reports on 126 young women attending the service over a five-year period. The primary aim was assessing the rate of women with diminished ovarian reserve (DOR) who may benefit from oocytes cryopreservation. 


\section{Materials and Methods}

This study took place at the Cancer Center IRCCS Istituto Nazionale dei Tumori (Milan, Italy). Since 2015, childhood and adolescent female tumor survivors are routinely offered fertility counseling at the oncofertility service of the hospital. This service takes place once a week and was originally implemented (in 2011) to discuss fertility preservation issues at the time of cancer diagnosis. Subsequently, it progressively broadened its activities, including post-tumor counseling. The service is run by a gynecologist who is expert in reproductive medicine (consultant, working in an external Infertility Unit of the same town - Fondazione IRCCS Ca' Granda Ospedale Maggiore Policlinico) and a gynecologist who is expert in oncology (internal of the hospital). The Cancer Center attracts patients from the whole country. The pediatric service that refers the patients to the oncofertility service is mostly engaged in the treatment of solid tumors. Pediatric hematological cancers are conversely generally treated by another Institution located in the same town.

Patients referred between January 2015 and June 2020 to the oncofertility service of the hospital were retrospectively reviewed. Inclusion criteria for the present study were as follows: 1) past history of tumors prior to 21 years (most were malignancies but we included also cases of recurrent ovarian dermoids), 2) gonadotoxic treatments of cancer (including chemotherapy, radiotherapy or ovarian surgery), 3) age $\geq 14$ years at referral, 4) at least one year from the end of oncologic treatments, 5) intact uterus. Subjects who did not return to provide the results of the prescribed ovarian tests within one year were considered non-adherent and were excluded. The study was approved by the local Ethical Committee.

Data regarding previous cancer treatments, menarche and menstrual cycle characteristics were collected. Alkylating agents exposure was presented as a dichotomous variable and using cyclophosphamide equivalent dose (CED) (13). These two variables do not fully correspond because dacarbazine, that is a non-classical alkylating agent, is not included in the CED formulae. Evaluation of the residual ovarian reserve was based on serum hormonal assessments [Follicle 
Stimulating Hormone (FSH), estradiol (E2)] on the second-third day of the menstrual cycle and Anti-mullerian Hormone (AMH)] and ultrasound evaluation of Antral Follicle Count (AFC). If under estroprogestin therapy, women were asked to preliminarily discontinue therapy for at least three months. Tests were not systematically performed for all referred subjects but tailored to the diagnostic purposes and the clinical situation. In particular, AFC was not assessed in subjects who did not have previous sexual intercourses, AFC and AMH were not deemed necessary in women with amenorrhea and serum FSH $>40 \mathrm{IU} / \mathrm{mL}$ and serum FSH was not considered essential if AFC and AMH were adequate. Within normal range serum FSH was deemed unreliable if E2 exceeded $80 \mathrm{pg} / \mathrm{mL}$. Subjects were invited to refer regularly after the first assessment to monitor the situation. Only data from the last assessment was reported in this study.

Primary amenorrhea was defined as the absence of menstrual flow by age 14 in the absence of growth or development of secondary sexual characteristics or by the age of 16 regardless of the presence of growth or development of secondary sexual characteristics (14). Secondary amenorrhea was the absence of menstrual cycles for 3 months in subjects who had regular menstrual cycles and 6-9 months for those with irregular menstrual cycles. Amenorrhea could be hyper-gonadotropic (primary gonadal failure) or hypo-gonadotropic (pituitary gonadotropin deficiency). Premature ovarian insufficiency (POI) was defined as premature exhaustion of the ovarian reserve and was diagnosed in women with amenorrhea and repeated peripheral levels of FSH above $40 \mathrm{IU} / \mathrm{mL}$ (14). A diagnosis of diminished ovarian reserve (DOR) was done when AMH or AFC were below the 5$10^{\text {th }}$ percentile of the age-related normograms $(15,16)$. Given the young age of the cohort, these thresholds approximately corresponded to serum AMH $<2 \mathrm{ng} / \mathrm{ml}(<1 \mathrm{ng} / \mathrm{ml}$ if the woman had one damaged or removed ovary) or total AFC $\leq 10(\leq 5$ if the woman had one damaged or removed ovar). Overt DOR was diagnosed in case of irregular cycles and severely compromised ovarian reserve tests $(\mathrm{AMH}<0.5 \mathrm{ng} / \mathrm{ml}$ and total $\mathrm{AFC}<5$ and $\mathrm{FSH}>15 \mathrm{IU} / \mathrm{mL})$. These latter cutoff points refer to the lower values suggested in the Bologna criteria (17). 
Young women were informed about the clinical significance of ovarian reserve. In case of DOR, they were told that a low ovarian reserve does not affect the chances of natural pregnancy if they are in the condition to seek for pregnancy in the short term and provided that the remnant ovarian reserve is sufficient to ensure regular menstrual cycles. However, future chances of pregnancy may be compromised if they postpone pregnancy seeking for several years (18-20). Although ovarian reserve measurements are not precise predictors of the duration of the fertile period, they currently offer the best approximation available $(21,22)$. Patients were informed about this uncertainty. They were also informed about alternative modes of conceptions such as oocytes donation and adoption, in particular for those who had severe impairment of the ovarian reserve.

Oocytes cryopreservation was considered in subjects aged $\geq 18$ years who were diagnosed with DOR. A shared decision-making approach was adopted. The patients were informed regarding the technique and the risks of oocytes cryopreservation (in particular the risks of hemorrhage and infection) (23). They were explained that the higher was the number of oocytes retrieved, the higher were the chances to obtain a pregnancy at the time of thawing and that the program was deemed sufficient or satisfactory if at least 10 or 20 oocytes could be stored, respectively $(24,25)$. Women were offered up to three attempts of ovarian hyperstimulation and oocytes retrieval. A protocol with Gonadotropin Releasing Hormone $(\mathrm{GnRH})$ antagonists was systematically used. The precise modalities of cycle monitoring and oocytes retrieval are explained in details elsewhere (11).

Oocytes cryopreservation procedure was performed as previously reported $(26,27)$. Briefly, after transvaginal ultrasound-guided aspiration and recovery, oocytes were incubated for $2-8 \mathrm{~h}$ in commercial culture medium and metaphase II (MII) oocytes were selected under a stereomicroscope. Vitrification was performed by using the Cryotop device (Kitazato Co., Fujinomiya, Japan) consisting of a thin film strip attached to a plastic holder and a separate protective cap to avoid mechanical damage of the strip during manipulation and storage. The first equilibration was performed in $7.5 \%$ ethylene glycol (EG) and 7.5\% dimethylsulfoxide (DMSO) at 
$24-26^{\circ} \mathrm{C}$ for $12-15 \mathrm{~min}$. Subsequently, oocytes were transferred to a $24-26^{\circ} \mathrm{C} 15 \% \mathrm{EG}, 15 \%$ DMSO and $0.5 \mathrm{M}$ sucrose solution for $1 \mathrm{~min}$, then placed on the film strip of the Cryotop as a single small drop. The excess solution was removed and the Cryotop was submerged into liquid nitrogen. The strip was covered with the protective cap and the sample was stored submerged in liquid or in vapor phase nitrogen. No important changes were made in the vitrification protocol during the investigation period. Overall, during the study period, the survival rate, the fertilization and the rate of embryo development were $71 \%$ per thawed oocytes, $54 \%$ per thawed oocytes $(76 \%$ per used oocytes) and $47 \%$ per thawed oocytes ( $87 \%$ per fertilized oocytes).

Costs were covered by the national public health system. Again, the counseling was tailored on the estimated residual ovarian reserve (the lower was the estimated remnant ovarian reserve, the lower were the chances of obtaining 10-20 oocytes). During the discussion with the woman regarding pros and cons of the procedure, the potential benefits were tailored to the magnitude of ovarian reserve impairment. Women with overt DOR were discouraged but not excluded.

Data is reported as number $(\%)$, mean $\pm \mathrm{SD}$ or median [interquartile range-IQR]. Only descriptive statistics were used. Data was analyzed with the Statistical Package for Social Sciences 23.0 (SPSS, IL, USA). No attempts were made to identify risk factors for ovarian reserve impairment because of the relatively low number of included subjects. However, the main outcomes (rate of women with preserved ovarian reserve, DOR, POI and oocytes storage) were reported separately for the subgroups of women at highest risk of ovarian reserve impairment. Specifically, data were presented disjointedly for women who were treated also for a recurrence or a second primary neoplasm, for those who received alkylating agents, those who had a CED $\geq 8$, those who underwent autologous hematopoietic stem cell transplant, those who had abdominal or pelvic radiotherapy and those who underwent gynecological surgery. The 95\% Confidence Interval (95\% CI) of proportions was calculated using a binomial distribution model: this estimate was reported only for the most relevant proportions. Based on the activity of the service during the study 
period, we calculated that our sample size (about 90 women) could provide amplitudes of the $95 \%$ CI within $20 \%$ ( $\pm 10 \%$ ) for rates of women performing oocytes retrieval lower than $40 \%$.

\section{Results}

One hundred twenty-six subjects were initially evaluated, of whom 90 could be included in this study (Supplemental Figure 1). Specifically, 26 out of 126 subjects (21\%) did not complete the assessment and were considered non-adherent. Ten additional cases were excluded because they were younger than 14 years of age $(n=5)$ or because they had not yet returned to complete the evaluation $(n=5)$.

Baseline characteristics of the selected young adult tumor survivors $(n=90)$ are shown in Table 1. The vast majority of the included subjects had malignancies, only one case was diagnosed with recurrent ovarian mature teratomas. Chemotherapy was not required in two cases. These two women who were exclusively treated with ovarian surgery (the woman with recurrent mature teratomas and one woman with immature teratoma). Chemotherapy was required in the remaining 88 cases $(98 \%)$. Fifty-nine different combinations of chemotherapeutic drugs were used. The most common were Bleomycin-Cisplatin-Etoposide $(n=12)$, Adriamycyn-Bleomycin-DacarbazinVinblastine ( $n=8)$, Cisplatin-Etoposide $(n=4)$, and Cisplatin-Lomustine-Vincristine $(n=3)$. Six other combinations were used in two cases per group, while the remaining 49 combinations were used only once.

Cycle characteristics and ovarian reserve testing are shown in Table 2. Twenty-three patients had amenorrhea, of whom 13 had primary and 10 had secondary amenorrhea. In 19 of these cases, amenorrhea was due to ovarian reserve exhaustion while in the remaining four it was due to central nervous system damage (hypogonadotropic hypogonadism). All these four cases had primary amenorrhea and ovarian reserve testing were not indicative of POI. 
Overall, we documented 19 cases of POI ( 9 with primary amenorrhea and 10 with secondary amenorrhea) and 35 cases of DOR. The corresponding proportions (95\%CI) were $21 \%(14-31 \%)$ and $39 \%(29-49 \%)$, respectively. In the remaining 36 young women (40\%, 95\%CI: 30-50\%), ovarian reserve was deemed adequate. The outcome of counseling in the subgroup of young women with DOR is summarized in Supplemental Table 1. Overall, 13 subjects were eligible for oocytes cryopreservation, of whom nine (69\%) performed the procedure. Considering the whole cohort of evaluated young women $(n=90)$, the rate of those who underwent egg freezing was $10 \%$ (95\%CI: 518\%). Main characteristics and cycle outcome of these nine women are shown in Supplemental Table 2. Frequencies of the main outcomes (rate of women with preserved ovarian reserve, DOR, POI and oocytes storage) in the subgroups of women at highest risk of ovarian reserve impairment are presented in Table 3. The rate of women with preserved ovarian reserve, DOR, and POI varied between $0 \%$ to $29 \%, 32 \%$ and $64 \%$, and $14 \%$ and $57 \%$, respectively. Egg freezing varied between $0 \%$ and $33 \%$. When considering the most common chemotherapeutic combinations, the number of women with preserved ovarian reserve, DOR, POI and those who underwent egg freezing were as follows: for Bleomycin-Cisplatin-Etoposide $(n=12)$, they were 5/6/1/2, for Adriamycyn-BleomycinDacarbazin-Vinblastine $(n=8)$ they were $7 / 1 / 0 / 1$, for Cisplatin-Etoposide $(n=4)$ they were 3/1/0/0, and for Cisplatin-Lomustine-Vincristine $(n=3)$ they were 1/2/0/0.

Finally, nine women initiated to seek for pregnancy after the first counseling. Excluding from the denominator those girls who were younger than 18 years of age and those with POI, this leads to a percentage of $17 \%$ ( 9 out of 54). If restricting to those older than 21 years, this percentage raises to $29 \%$ (9 out of 31). Overall, seven out of these nine women had a baby or an ongoing pregnancy at the last assessment. One of these women had to turn to in vitro fertilization (IVF) (Supplemental Table 3). One woman underwent two IVF cycles without success (in both occasions ovarian response was modest but sufficient to ensure the transfer of 1-2 embryos), the other one interrupted natural pregnancy seeking for personal reasons after one year. 


\section{Discussion}

A consistent proportion of young survivors referring for fertility assessment and pro-fertility counseling were found to have ovarian reserve impairment. POI was diagnosed in $21 \%$ of women $(n=19)$ while $39 \%(n=35)$ had a clinically relevant reduction of the ovarian reserve (DOR). Noteworthy, in 8 out of these 35 cases (23\%), ovarian reserve was severely compromised (overt DOR). Overall, one in ten women in our cohort performed oocytes cryopreservation $(10 \%, 95 \% \mathrm{CI}$ : $5-18 \%)$. To note, this percentage underestimates the acceptability of the procedure. Indeed, we did not suggest oocytes cryopreservation to all women, but only to those aged $\geq 18$ years with DOR (and discouraging those with overt DOR). In fact, only four out of 13 eligible women declined the procedure. The acceptance rate was thus $69 \%$ ( 9 out of 13). This is remarkable considering the wellknown emotional and physical burden of ovarian hyper-stimulation and oocytes retrieval $(12,28,29)$.

The study is underpowered to draw indications on which subgroup of women would merit more attention. We could only highlight that those exclusively treated with Adriamycyn-BleomycinDacarbazin-Vinblastine may be less harmed. However, in general, we do not believe that particular selection criteria can be applied. In this regard, it is interesting to note that considerable differences emerged according to the therapeutic history of the women. Among women exposed to most gonadotoxic treatments, the rates of DOR, POI and egg freezing varied substantially (Table 3). However, the proportion of DOR women varied less (between $32 \%$ and 64\%) compared to preserved ovarian reserve and POI (that are conversely inversely correlated). This observation further strengthens the idea that all women deserve fertility counseling regardless of the specific treatment received.

Previous evidence on post-cancer fertility counseling in the literature is scant $(1,30)$. Evidence specifically focusing on the peculiar population of women who had a tumour in their childhood or 
adolescence is even more limited. We identified only two previous studies on this issue $(12,31)$. In the first one, Lehman et al. presented their experience on 80 young female survivors: preserved ovarian reserve, DOR and POI were diagnosed in $8(10 \%), 21(26 \%)$ and 51 (64\%) women, respectively. Eleven subjects with DOR (52\%) stored their eggs. The rate of eligible women declining oocytes cryopreservation was 5\% (12). The second study illustrated ovarian reserve status in 55 women previously treated for pediatric cancers but did not report on the possibility to freeze oocytes. Ovarian reserve was compromised in 23 (42\%) patients: 14 with DOR and 9 with an established POI (31). Overall, results from these two studies are in line with our findings. Nonetheless, some mild differences emerged. They may be explained by statistical fluctuations (in all three studies the sample size was not large), the thresholds used to define DOR, the type of counseling and the baseline characteristics of the studied cases. For instance, in our setting, hematological cases were mainly referred to a dedicated independent hospital, and thus are inevitably under-represented. Moreover, the vast majority of women received chemotherapy with alkylating agents $(61 \%)$ or needed radiotherapy (61\%), one fourth had autologous hematopoietic stem cell transplant $(23 \%)$ and one third had a recurrence or a second primary neoplasm (20\% and $14 \%$, respectively), suggesting that some selection biases towards more injured cases may have occurred.

Childhood tumor survivors are an ever-growing population among which questions and the interest on the long term effects of cancer treatment has grown in recent years $(1,8)$. Nowadays, there is a general consensus that quality of survival needs the same attention as the quantity of survival (1). In line with this evolution of cancer management, the vision of fertility preservation for young patients with cancer is also changing. Providing fertility preservation prior to treatment initiation is important, but addressing fertility across the cancer continuum is crucial $(12,32)$. Of utmost relevance is that tumor survivors are generally upset by the possibility that their fertility can be hampered (12)- but they tend to hide their concerns for years, usually until they approach childbearing age and start forming romantic relationships. Therefore, promptly investigating and 
facing these uncertainties seems essential. Accordingly, adherence to our program was high, as shown by the low rate of those who did not complete the requested investigations. Despite the wellknown clinical over-burden of tumor survivors and the difficulties related to referral setting (subjects were referred from all over the country), only one out of five did not return to complete the counseling (26 out of 126 subjects, corresponding to $21 \%$ ).

Physicians should not be afraid of causing additional harm, as ignoring fertility concerns may be worse for psychological well-being of the patients. A complete and realistic counseling on the residual ovarian reserve is essential and can empower young women. For instance, they may decide to modify their reproductive plans by anticipating motherhood or may undergo egg freezing. To note, also young women with POI deserves to be counseled by physicians engaged in reproductive medicine. Oocytes donation is a valuable treatment for these women, but this option is frequently misperceived by the general population $(33,34)$. A clear and realistic information is warranted to mitigate anxiety and to prevent mis-perceptions on their future chances of motherhood.

The idea to provide fertility counseling for the general population was first claimed by Hvidman et al. in 2015 (35), and since then it has raised growing interest (1). However, this initiative has also created debate (36). The main concern is the risk of causing anxiety and over-treatment in the absence of a robust evidence (36,37). In particular, the interpretation of assessments suggesting reduced ovarian reserve remains challenging. A poor result could have potentially devastating psychological and social sequelae, and for this reason we deem crucial providing reliable information (37). On one hand autonomy and personal empowerment represent a cornerstone of medical ethics, on the other hand providing unreliable or imprecise information could cause harm. According to a recent individual patient data meta-analysis, only $28 \%$ of women with AMH below the fifth percentile enter menopause before 40 years of age (21). To note, early menopause can be detrimental from a hormonal and general health perspective but may not cause childlessness if women do not excessively postpone pregnancy seeking (38). The end of fertility is estimated to be 
around 41 years (39) and entering into menopause around this age cannot be expected to markedly affect the overall chances of childbearing. In agreement with this view, low AMH was repeatedly demonstrated to have no effect on the chances of natural pregnancy (18-20). This is also in line with our data that showed that most women who attempted to become pregnant succeeded. To note, four women who got pregnant had DOR, of whom one had overt DOR. More in general, current knowledge on ovarian reserve is incomplete. We have insufficient evidence to draw a personal trajectory of ovarian reserve loss that could help us to accurately predict the duration of the fertile period $(22,40)$. Counseling must handle this uncertainty, finding out an equilibrium between inopportune reassurance and unproven grim predictions. Monitoring ovarian tests over time may be more informative but data on the validity of this approach is lacking.

Some main limitations of our study should be acknowledged. Firstly, we did not assess the psychological well-being or the rate of satisfaction of the included women. We relied on the literature that strengthens the importance of a comprehensive and continuum care but we do not provide direct information. To note, a comprehensive fertility counseling cannot be limited to ovarian reserve assessment. Other dimensions such as sexuality and capacity to develop romantic relationships should be concomitantly investigated. Secondly, the decision to postpone oocytes cryopreservation after the age of 18 years could be a point of concern. One may argue that this can further reduce the residual ovarian reserve in case of severe damage. The remnant pool of primordial follicles progressively reduces with age and one may claim that performing egg freezing prior to reach 18 years (i.e. at 15-16 years) could be more effective. On the other hand, we deemed inappropriate to anticipate fertility preservation techniques to minors for ethical reasons (41). In minors, it is arguable to perform any treatment that can be postponed because the consent is obtained by relatives and not directly by the patients themselves. In addition, it has recently been shown that oocytes competence could be altered in very young girls (42). Thirdly, our selection criteria allowed the inclusion of non-cancer patients. In fact, only two young women with recurrent dermoids were included. Even if inevitably poorly informative, these cases highlighted the 
importance of a broader inclusion of patients. Non-malignant cases may also be severely concerned by fertility issues. Fourthly, we have to acknowledge that only four girls (4\%) in our cohort underwent ovarian cortex freezing. This rate is inappropriately low and may influence the high adherence observed in our study. To note, this low rate does not reflect the actual current practice. Most patients included in this cohort had a diagnosis of cancer 10-20 years ago when the procedure was still highly experimental and unavailable in our setting. The situation is expected to rapidly improve over the next years. Finally, we did not adopt a stringent definition of DOR. The use of a single biomarker of ovarian reserve (either AMH or AFC) and a stringent threshold would have facilitated inferences and comparisons with other studies. On the other hand, biomarkers of ovarian reserve are imprecise and fluctuations are common. In addition, the accuracy of AMH remains debated in the pediatric and adolescent population $(43,44)$. For these reasons, we decided to adopt a more personalized and less rigid approach. The use of stringent cutoff points would have simplified the counseling but lacks scientifically validity.

At present, robust clinical recommendations cannot be drawn from our findings. We showed that ovarian reserve impairment is common, that oocytes cryopreservation is feasible, and that empowerment can lead to anticipate motherhood. However, we did not collect data on women satisfaction, neither perform cost-beneficial analyses, thus limiting our capacity for stringent recommendations. On the other hand, given the ethical value of patients' empowerment (36), we plea at least for a systematic referral of childhood and adolescent female tumor survivors to reproductive specialists for an in-depth and personalized counseling. This recommendation is particularly valid once women are aged $\geq 18$ years, but in selected cases it can be considered earlier. Conversely, there is insufficient evidence to strongly recommend oocytes cryopreservation in these women. Additional evidence is needed to determine the real benefits of this program, and to identify the precise characteristics of women who should be candidate. 
In conclusion, post-cancer oocytes cryopreservation is an emerging opportunity that may deserve consideration within the complex and multifaceted armamentarium of fertility preservation options. It should not be viewed as an alternative to fertility preservation at the time of cancer diagnosis but as an additional option. We advocate a systematic referral of childhood and adolescent female tumor survivors once they are $\geq 18$ years This would allow all women to empower themselves on their reproductive potential and could give an additional chance of motherhood in some of them. However, there are some areas of uncertainty that can be challenging, in particular the interpretation of ovarian reserve tests. In our opinion, these uncertainties should be acknowledged and handled using a shared and tailored decision-making approach. To note, there is in general no need for a prompt and definite decision and the adolescent and young woman can be accompanied over the years. Decisions such as egg freezing or anticipating pregnancy seeking may require time and need to be progressively settled. 


\section{References}

1. Macklon KT, Fauser Cjm B. The female post-cancer fertility-counselling clinic: looking beyond the freezer. A much needed addition to oncofertility care. Reprod Biomed Online. 2019; 39: 179-181.

2. Anazodo A, Laws P, Logan S, Saunders C, Travaglia J, Gerstl B, Bradford N, Cohn R, Birdsall M, Barr R, Suzuki N, Takae S, Marinho R, Xiao S, Qiong-Hua C, Mahajan N, Patil M, Gunasheela D, Smith K, Sender L, Melo C, Almeida-Santos T, Salama M, Appiah L, Su I, Lane S, Woodruff TK, Pacey A, Anderson RA, Shenfield F, Ledger W, Sullivan E. How can we improve oncofertility care for patients? A systematic scoping review of current international practice and models of care. Hum Reprod Update. 2019; 25: 159-179.

3. Anazodo AC, Choi S, Signorelli C, Ellis S, Johnston K, Wakefield CE, Deans R, Neville KA, Cohn RJ. Reproductive Care of Childhood and Adolescent Cancer Survivors: A 12-Year Evaluation. J Adolesc Young Adult Oncol. 2020 Nov 11 [Epub ahead of print].

4. Newton K, Howard AF, Thorne S, Kelly MT, Goddard K. Facing the unknown: uncertain fertility in young adult survivors of childhood cancer. J Cancer Surviv. 2020 Jul 1. [Epub ahead of print].

5. van Santen HM, van de Wetering MD, Bos AME, Vd Heuvel-Eibrink MM, van der Pal HJ, Wallace WH. Reproductive Complications in Childhood Cancer Survivors. Pediatr Clin North Am. 2020; 67: 1187-1202.

6. Crawshaw MA, Sloper P. 'Swimming against the tide'--the influence of fertility matters on the transition to adulthood or survivorship following adolescent cancer. Eur J Cancer Care (Engl). 2010; 19: 610-620.

7. Terenziani M, Meazza C, Massimino M, Viganò P, Gandola L, Mangili G, Raspagliesi F, Biasoni D, Podda M, Veneroni L, Filippi F, Riccipetitoni G, Somigliana E. Female fertility preserving practices at a pediatric unit: a challenge of multiprofessional and multidisciplinary cooperation. Tumori. 2016; 102: 174-177.

8. Fidler MM, Frobisher C, Hawkins MM, Nathan PC. Challenges and opportunities in the care of survivors of adolescent and young adult cancers. Pediatr Blood Cancer. 2019; 66: e27668. 
9. Lehmann V, Chemaitilly W, Lu L, Green DM, Kutteh WH, Brinkman TM, Srivastava DK, Robison LL, Hudson MM, Klosky JL. Gonadal Functioning and Perceptions of Infertility Risk Among Adult Survivors of Childhood Cancer: A Report From the St Jude Lifetime Cohort Study. J Clin Oncol. 2019; 37: 893-902.

10. Young K, Shliakhtsitsava K, Natarajan L, Myers E, Dietz AC, Gorman JR, Martínez ME, Whitcomb BW, Su HI. Fertility counseling before cancer treatment and subsequent reproductive concerns among female adolescent and young adult cancer survivors. Cancer. 2019; 125: 980-989.

11. Filippi F, Meazza C, Paffoni A, Raspagliesi F, Terenziani M, Somigliana E. Egg Freezing in Childhood and Young Adult Cancer Survivors. Pediatrics. 2016; 138: e20160291.

12. Lehmann V, Kutteh WH, Sparrow CK, Bjornard KL, Klosky JL. Fertility-related services in pediatric oncology across the cancer continuum: a clinic overview. Support Care Cancer. 2020; 28: 3955-3964.

13. Green DM, Nolan VG, Goodman PJ, Whitton JA, Srivastava D, Leisenring WM, Neglia JP, Sklar CA, Kaste SC, Hudson MM, Diller LR, Stovall M, Donaldson SS, Robison LL. The cyclophosphamide equivalent dose as an approach for quantifying alkylating agent exposure: a report from the Childhood Cancer Survivor Study. Pediatr Blood Cancer. 2014; 61: 53-67.

14. Pastore LM, Christianson MS, Stelling J, Kearns WG, Segars JH. Reproductive ovarian testing and the alphabet soup of diagnoses: DOR, POI, POF, POR, and FOR. J Assist Reprod Genet. 2018; 35: 17-23.

15. Bozdag G, Calis P, Zengin D, Tanacan A, Karahan S. Age related normogram for antral follicle count in general population and comparison with previous studies. Eur $\mathrm{J}$ Obstet Gynecol Reprod Biol. 2016; 206: 120-124

16. Du X, Ding T, Zhang H, Zhang C, Ma W, Zhong Y, Qu W, Zheng J, Liu Y, Li Z, Huang K, Deng S, Ma L, Yang J, Jiang J, Yang S, Huang J, Wu M, Fang L, Lu Y, Luo A, Wang S. Age-Specific Normal Reference Range for Serum Anti-Müllerian Hormone in Healthy Chinese Han Women: A nationwide Population-Based Study. Reprod Sci. 2016; 23:10191027 
17. Ferraretti AP, La Marca A, Fauser BC, Tarlatzis B, Nargund G, Gianaroli L; SHRE working group on Poor Ovarian Response Definition. ESHRE consensus on the definition of 'poor response' to ovarian stimulation for in vitro fertilization: the Bologna criteria. Hum Reprod. 2011; 26: 1616-1624

18. Somigliana E, Lattuada D, Colciaghi B, Filippi F, La Vecchia I, Tirelli A, Baffero GM, Paffoni A, Persico N, Bolis G, Fedele L. Serum anti-Müllerian hormone in subfertile women. Acta Obstet Gynecol Scand. 2015; 94: 1307-1312.

19. Zarek SM, Mitchell EM, Sjaarda LA, Mumford SL, Silver RM, Stanford JB, Galai N, White MV, Schliep KC, DeCherney AH, Schisterman EF. Is Anti-Müllerian Hormone Associated With Fecundability? Findings From the EAGeR Trial. J Clin Endocrinol Metab. 2015; 100: 4215-4221.

20. Steiner AZ, Pritchard D, Stanczyk FZ, Kesner JS, Meadows JW, Herring AH, Baird DD. Association Between Biomarkers of Ovarian Reserve and Infertility Among Older Women of Reproductive Age. JAMA. 2017; 318: 1367-1376.

21. Depmann M, Eijkemans MJC, Broer SL, Tehrani FR, Solaymani-Dodaran M, Azizi F, Lambalk CB, Randolph JF Jr, Harlow SD, Freeman EW, Sammel MD, Verschuren WMM, van der Schouw YT, Mol BW, Broekmans FJM. Does AMH relate to timing of menopause? Results of an Individual Patient Data meta- analysis. J Clin Endocrinol Metab. 2018; 103: 3593-3600.

22. Somigliana E, Terenziani M, Filippi F, Bergamini A, Martinelli F, Mangili G, Peccatori F, Vercellini P. Chemotherapy-related damage to ovarian reserve in childhood cancer survivors: interpreting the evidence. J Assist Reprod Genet. 2019; 36: 341-348.

23. Schattman GL. CLINICAL PRACTICE. Cryopreservation of Oocytes. N Engl J Med. 2015; 373: $1755-1760$.

24. Goldman RH, Racowsky C, Farland LV, Munné S, Ribustello L, Fox JH. Predicting the likelihood of live birth for elective oocyte cryopreservation: a counseling tool for physicians and patients. Hum Reprod. 2017; 32: 853-859.

25. Cobo A, García-Velasco J, Domingo J, Pellicer A, Remohí J. Elective and Onco-fertility preservation: factors related to IVF outcomes. Hum Reprod. 2018; 33: 2222-2231. 
26. Kuwayama M, Vajta G, Kato O, Leibo SP. Highly efficient vitrification method for cryopreservation of human oocytes. Reprod Biomed Online. 2005; 11: 300-308.

27. Rienzi L, Cobo A, Paffoni A, Scarduelli C, Capalbo A, Vajta G, Remohí J, Ragni G, Ubaldi FM. Consistent and predictable delivery rates after oocyte vitrification: an observational longitudinal cohort multicentric study. Hum Reprod. 2012; 27: 1606-1612.

28. Gameiro S, Boivin J, Peronace L, Verhaak CM. Why do patients discontinue fertility treatment? A systematic review of reasons and predictors of discontinuation in fertility treatment. Hum Reprod Update. 2012; 18: 652-669.

29. Gameiro S, Verhaak CM, Kremer JA, Boivin J. Why we should talk about compliance with assisted reproductive technologies (ART): a systematic review and meta-analysis of ART compliance rates. Hum Reprod Update. 2013; 19: 124-135.

30. Massarotti C, Scaruffi P, Lambertini M, Sozzi F, Remorgida V, Anserini P. Beyond fertility preservation: role of the oncofertility unit in the reproductive and gynecological follow-up of young cancer patients. Hum Reprod. 2019; 34: 1462-1469.

31. Parissone F, Di Paola R, Balter R, Garzon S, Zaffagnini S, Neri M, Vitale V, Tridello G, Cesaro S. Female adolescents and young women previously treated for pediatric malignancies: assessment of ovarian reserve and gonadotoxicity risk stratification for early identification of patients at increased infertility risk. J Pediatr Endocrinol Metab. 2020 [Epub ahead of print].

32. Roeca C, Dovey S, Polotsky AJ. Recommendations for assessing ovarian health and fertility potential in survivors of childhood cancer. Maturitas. 2019; 122: 57-59.

33. Canzi E, Accordini M, Facchin F. 'Is blood thicker than water?' Donor conceived offspring's subjective experiences of the donor: a systematic narrative review. Reprod Biomed Online. 2019; 38: 797-807.

34. Skoog Svanberg A, Sydsjö G, Lampic C. Psychosocial aspects of identity-release gamete donation - perspectives of donors, recipients, and offspring. Ups J Med Sci. 2020; 125: 175182. 
35. Hvidman HW, Petersen KB, Larsen EC, Macklon KT, Pinborg A, Nyboe Andersen A. Individual fertility assessment and pro-fertility counselling; should this be offered to women and men of reproductive age? Hum Reprod. 2015; 30: 9-15.

36. Tremellen K, Savulescu J. Ovarian reserve screening: a scientific and ethical analysis. Hum Reprod. 2014; 29: 2606-2614.

37. O'Brien Y, Kelleher C, Wingfield M. "So what happens next?" exploring the psychological and emotional impact of anti-Mullerian hormone testing. J Psychosom Obstet Gynaecol. 2020; 41: 30-37.

38. Daan NM, Hoek A, Corpeleijn E, Eijkemans MJ, Broekmans FJ, Fauser BC, Koster MP. Reproductive characteristics of women diagnosed with premature ovarian insufficiency. Reprod BioMed Online. 2016; 32: 225-232.

39. Eijkemans MJ, van Poppel F, Habbema DF, Smith KR, Leridon H, te Velde ER. Too old to have children? Lessons from natural fertility populations. Hum Reprod. 2014; 29: 1304-1312.

40. Nielsen SN, Andersen AN, Schmidt KT, Rechnitzer C, Schmiegelow K, Bentzen JG, Larsen EC. A 10-year follow up of reproductive function in women treated for childhood cancer. Reprod Biomed Online. 2013; 27: 192-200.

41. Mertes H. Let's not forget that many prepubertal girls do have other options besides ovarian tissue cryopreservation. Hum Reprod. 2015; 30: 2011-2013

42. Gruhn JR, Zielinska AP, Shukla V, Blanshard R, Capalbo A, Cimadomo D, Nikiforov D, Chan AC, Newnham LJ, Vogel I, Scarica C, Krapchev M, Taylor D, Kristensen SG, Cheng J, Ernst E, Bjørn AB, Colmorn LB, Blayney M, Elder K, Liss J, Hartshorne G, Grøndahl ML, Rienzi L, Ubaldi F, McCoy R, Lukaszuk K, Andersen CY, Schuh M, Hoffmann ER. Chromosome errors in human eggs shape natural fertility over reproductive life span. Science. 2019; 365: 1466-1469.

43. Kelsey TW, Anderson RA, Wright P, Nelson SM, Wallace WH. Data-driven assessment of the human ovarian reserve. Mol Hum Reprod. 2012; 18: 79-87.

44. Jopling H, Yates A, Burgoyne N, Hayden K, Chaloner C, Tetlow L. Paediatric Anti-Müllerian Hormone measurement: Male and female reference intervals established using the automated Beckman Coulter Access AMH assay. Endocrinol Diabetes Metab. 2018; 1: e00021. 
Figure legend

Supplemental Figure 1: Flow-chart of the study

Cohort of referred patients

$\mathrm{n}=126$

Excluded $(n=36)$

- Age $<14$ years $(n=5)$

- Incomplete data $(n=26)$

- Ongoing evaluation ( $\mathrm{n}=5$ )

Completed ovarian reserve testing $\mathrm{n}=90$ 
Table 1. Baseline characteristics of the patients at the time of cancer diagnosis

\begin{tabular}{|c|c|}
\hline Characteristics & $\begin{array}{c}\mathrm{N}(\%) \text { or mean } \\
\pm \mathrm{SD}\end{array}$ \\
\hline Age at diagnosis (years) & $10.6 \pm 5.4$ \\
\hline \multicolumn{2}{|l|}{ Menarche } \\
\hline Pre-menarchal & $55(61 \%)$ \\
\hline Post-menarchal & $35(39 \%)$ \\
\hline \multicolumn{2}{|l|}{ Cancer diagnosis } \\
\hline Lymphomas & $15(17 \%)$ \\
\hline Kidney tumours & $6(7 \%)$ \\
\hline Bone sarcomas & $14(16 \%)$ \\
\hline Central nervous system & $22(24 \%)$ \\
\hline Neuroblastoma & $8(9 \%)$ \\
\hline Soft tissue sarcoma & $10(11 \%)$ \\
\hline Others & $15(17 \%)$ \\
\hline Recurrence & $18(20 \%)$ \\
\hline Second primary neoplasm & $13(14 \%)$ \\
\hline \multicolumn{2}{|l|}{ Chemotherapy } \\
\hline None & $2(2 \%)$ \\
\hline Non alkylating & $33(37 \%)$ \\
\hline Alkylating & $55(61 \%)$ \\
\hline \multicolumn{2}{|l|}{$\operatorname{CED}\left(\mathrm{g} / \mathrm{m}^{2}\right)$} \\
\hline None & $35(39 \%)$ \\
\hline$<4$ & $4(4 \%)$ \\
\hline 4 to $<8$ & $15(17 \%)$ \\
\hline$\geq 8$ & $36(40 \%)$ \\
\hline Autologous hematopoietic stem cell transplant & $21(23 \%)$ \\
\hline \multicolumn{2}{|l|}{ Radiotherapy } \\
\hline None & $35(39 \%)$ \\
\hline Central nervous system & $18(20 \%)$ \\
\hline Abdominal & $14(16 \%)$ \\
\hline Pelvic & $8(9 \%)$ \\
\hline Other sites & $15(17 \%)$ \\
\hline \multicolumn{2}{|l|}{ Surgery ${ }^{a}$} \\
\hline None & $31(34 \%)$ \\
\hline Central nervous system & $12(13 \%)$ \\
\hline Gynecological $^{\mathrm{b}}$ & $14(16 \%)$ \\
\hline Others & $33(37 \%)$ \\
\hline \multicolumn{2}{|l|}{ Fertility preservation } \\
\hline Ovarian cortex freezing & $4(4 \%)$ \\
\hline GnRH analogues & $10(11 \%)$ \\
\hline
\end{tabular}


Data refers to the whole therapeutic journey (i.e. including treatments needed to cure recurrences or second tumours).

${ }^{a}$ If women underwent more than one surgery only the most detrimental to reproductive capacity is reported.

${ }^{b}$ Include 14 women who performed unilateral cystectoomy $(n=2)$, unilateral ovariectomy $(\mathrm{n}=7)$, unilateral ovariectomy and contralateral cystectomy $(n=4)$ and bilateral adnexectomy $(n=1)$.

CED: Cyclophosphamide equivalent dose 
Table 2. Characteristics of the women who completed the assessment ( $\mathrm{n}=90)$.

\begin{tabular}{cc}
\hline \multicolumn{1}{c}{ Characteristics } & $\begin{array}{c}\text { N } \% \text { ) or mean } \\
\pm \text { SD }\end{array}$ \\
\hline $\begin{array}{c}\text { Age at last referral (years) } \\
\text { Menarche }\end{array}$ & $21.3 \pm 5.4$ \\
Primary amenorrea & $13(14 \%)$ \\
Age (years) & $12.3 \pm 1.2$ \\
Hypogonadotropic hypogonadism & $4(4 \%)$ \\
Menstrual cycles a & \\
Amenorrhea & $23(26 \%)$ \\
$<23$ days & $3(3 \%)$ \\
$23-35$ days & $47(52 \%)$ \\
$>35$ days & $17(19 \%)$ \\
Hormonal therapy & $12(13 \%)$ \\
HRT & $19(21 \%)$ \\
Oral contraceptives & \\
AMH (ng/ml) & $23(26 \%)$ \\
$<0.5$ & $24(27 \%)$ \\
$0.5-2.0$ & $26(29 \%)$ \\
$>2.0$ & $17(19 \%)$ \\
Not available & \\
Antral Follicle Count (AFC) per ovary & $21(23 \%)$ \\
$<5$ & $21(23 \%)$ \\
$5-10$ & $17(19 \%)$ \\
$>11$ & $31(34 \%)$ \\
Not available & \\
FSH (IU/mL) & $37(41 \%)$ \\
$10-39$ & $30(10 \%)$ \\
$\geq 40$ & \\
Not available & \\
& \\
\hline & \\
\hline &
\end{tabular}

${ }^{\text {a }}$ Women assuming contraception were requested to discontinue the therapy for at least three months to assess the regularity of the cycle and to test ovarian reserve 
Table 3. Ovarian reserve status according to the most potentially harmful clinical conditions

\begin{tabular}{|c|c|c|c|c|c|}
\hline & $\mathrm{N}$. & $\begin{array}{c}\text { Preserved } \\
\text { ovarian reserve }\end{array}$ & DOR & POI & $\begin{array}{l}\text { Oocytes } \\
\text { cryostorage }\end{array}$ \\
\hline Recurrence or second primary neoplasm & 29 & $4(14 \%)$ & $15(52 \%)$ & $10(34 \%)$ & $4(14 \%)$ \\
\hline Use of alkylating agents & 55 & $16(29 \%)$ & $24(44 \%)$ & $15(27 \%)$ & $4(7 \%)$ \\
\hline $\mathrm{CED} \geq 8$ & 36 & $7(19 \%)$ & $18(50 \%)$ & $11(31 \%)$ & $3(8 \%)$ \\
\hline Autologous hematopoietic stem cell transplant & 21 & $0(0 \%)$ & $9(43 \%)$ & $12(57 \%)$ & $2(10 \%)$ \\
\hline Abdominal or pelvic radiotherapy & 22 & $5(23 \%)$ & $7(32 \%)$ & $10(45 \%)$ & $0(0 \%)$ \\
\hline Gynecological surgery & 14 & $3(22 \%)$ & $9(64 \%)$ & $2(14 \%)$ & $3(21 \%)$ \\
\hline All cohort & 90 & $36(40 \%)$ & $35(39 \%)$ & $19(21 \%)$ & $9(10 \%)$ \\
\hline
\end{tabular}

DOR: diminished ovarian reserve. POI: Premature Ovarian Insufficiency. CED: Cyclophosphamide equivalent dose All percentages refer to the total number of women 
Supplemental Table 1. Outcomes in women with DOR $(\mathrm{n}=35)$

\begin{tabular}{lc}
\hline \multicolumn{1}{c}{ Outcome } & $\mathrm{N}(\%)$ \\
\hline Oocytes retrieval performed & $9(26 \%)$ \\
Postponed (minor) & $8(23 \%)$ \\
Overt DOR & $8(23 \%)$ \\
Contraindication to pregnancy & $1(3 \%)$ \\
Death & $1(3 \%)$ \\
Seek natural conception ${ }^{\text {a }}$ & $4(11 \%)$ \\
Declined / not returned & $4(11 \%)$ \\
\hline
\end{tabular}

${ }^{a}$ In addition, one woman with overt DOR and one who banked her oocytes started seeking pregnancy (total 6 cases). 
Supplemental Table 2. Cycle outcome of women who cryopreserved their oocytes

\begin{tabular}{clcccccc}
\hline $\begin{array}{c}\text { Patient } \\
\text { N. }\end{array}$ & \multicolumn{1}{c}{ Diagnosis } & $\begin{array}{c}\text { Age } \\
\text { (years) }\end{array}$ & $\begin{array}{c}\text { FSH } \\
(\mathrm{IU} / \mathrm{ml})\end{array}$ & $\begin{array}{c}\text { AMH } \\
(\mathrm{ng} / \mathrm{ml})\end{array}$ & AFC & N. cyles & $\begin{array}{c}\text { N. stored } \\
\text { oocytes }\end{array}$ \\
\hline 1 & Glyoblastoma & 18 & 13.3 & 0.2 & 4 & 2 & 5 \\
2 & Ovarian germ cell tumour & 23 & 6.7 & 1.4 & 9 & 3 & 14 \\
3 & Hodgkin Linfoma & 20 & 4.4 & 0.5 & 3 & 1 & 7 \\
4 & Recurrent mature ovarian teratoma & 19 & 7.6 & 0.9 & 10 & 1 & 9 \\
5 & Soft tissue sarcoma & 26 & 5.4 & 0.5 & 7 & 3 & 17 \\
6 & Glyoblastoma & 21 & 12.5 & 0.2 & 7 & 2 & 7 \\
7 & Medulloblastoma & 25 & 13 & 0.2 & 5 & 2 & 1 \\
8 & Hodgkin Linfoma and soft tissue sarcoma & 23 & 7.6 & 0.9 & 5 & 3 & 19 \\
9 & Ovarian germ cell tumour & 25 & 6.8 & 1.7 & 5 & 1 & 16 \\
\hline
\end{tabular}

Patient N. 7 started seeking pregnancy after these two disappointing attempts and had a natural pregnancy. AFC: Antral Follicle Count 
Supplemental Table 3. Outcome of the women who decided to seek for pregnancy

\begin{tabular}{|c|c|c|c|c|c|c|c|}
\hline $\begin{array}{l}\text { Patient } \\
\text { N. }\end{array}$ & Diagnosis & $\begin{array}{c}\text { Age } \\
\text { (years) }\end{array}$ & $\begin{array}{c}\text { FSH } \\
(\mathrm{IU} / \mathrm{ml})\end{array}$ & $\begin{array}{c}\mathrm{AMH} \\
(\mathrm{ng} / \mathrm{ml})\end{array}$ & $\mathrm{AFC}$ & $\begin{array}{l}\text { Time to pregnancy } \\
\text { (months) }\end{array}$ & ART \\
\hline 1 & Wilms Tumor & 26 & 31.0 & 0.1 & 3 & 12 & No \\
\hline 2 & Osteosarcoma & 32 & 5.9 & 0.8 & 7 & 24 & $\operatorname{IVF}(n=2$ \\
\hline 3 & Medulloblastoma & 26 & 13.0 & 0.2 & 5 & 1 & No \\
\hline 4 & Dysgerminoma and Yolk sac ovarian neoplasia & 30 & 15.1 & 0.5 & 3 & 24 & $\operatorname{IVF}(n=2$ \\
\hline 5 & Malignant peripheral nerve sheath tumor & 33 & 11.2 & 0.2 & 4 & 2 & No \\
\hline 6 & Hodgkin Lymphoma & 30 & 12.0 & 4.0 & 20 & 6 & No \\
\hline 7 & Ewing Sarcoma & 26 & 5.5 & 4.1 & 2 & 3 & No \\
\hline 8 & Osteosarcoma & 29 & 3.8 & 1.0 & 8 & 1 & No \\
\hline 9 & Wilms Tumor & 29 & 4.8 & 4.9 & 30 & 4 & No \\
\hline
\end{tabular}

Patient N.1 stopped seeking pregnancy after one year for personal reasons. Patient N. 7 had two live births. 